\title{
Efficient computation of matched solutions of the Kapchinskij-Vladimirskij envelope equations for periodic focusing lattices
}

\author{
Steven M. Lund* \\ Lawrence Livermore National Laboratory, Livermore, California 94550, USA \\ Sven H. Chilton and Edward P. Lee \\ Lawrence Berkeley National Laboratory, Berkeley, California 94720, USA \\ (Received 13 January 2006; published 20 June 2006)
}

\begin{abstract}
A new iterative method is developed to numerically calculate the periodic, matched beam envelope solution of the coupled Kapchinskij-Vladimirskij equations describing the transverse edge trajectory of a beam in a periodic, linear focusing lattice of arbitrary complexity. Implementation of the method is straightforward. It is highly convergent and can be applied to all usual parametrizations of the matched envelope solutions. The method is applicable to all classes of linear focusing lattices without skew couplings, and also applies to all physically achievable system parameters - including cases where the matched beam envelope is strongly unstable. Example applications are presented for periodic solenoidal and quadrupole focusing lattices. Convergence properties are summarized over a wide range of system parameters.
\end{abstract}

DOI: 10.1103/PhysRevSTAB.9.064201

\section{INTRODUCTION}

The Kapchinskij-Vladimirskij (KV) envelope equations [1-3] are often employed as a simple model of the transverse evolution of intense ion beams. The equations are coupled ordinary differential equations that describe the evolution of the beam edge (or rms radii) in response to applied linear focusing forces of the lattice and defocusing forces resulting from beam space-charge and transverse phase-space area (emittances). Although the KV envelope equations are only fully Vlasov consistent with the singular $\mathrm{KV}$ distribution, the equations can be applied to describe the low-order evolution of a real distribution of beam particles when the variation of the statistical beam emittances is negligible or sufficiently slow [2]. Nonlinear fields that can be produced by nonideal applied focusing elements, nonuniform beam space-charge, and species contamination (electron cloud effects, etc.) drive deviations from the KV model. Such effects are suppressed to the extent possible in most practical designs, rendering the KV model widely applicable.

The matched solution of the KV envelope equations is the solution with the same periodicity as the focusing lattice [1-3]. The matched beam envelope is important because it is believed to be the most radially compact solution supported by a periodic linear focusing channel [3]. Matched envelopes are typically calculated as a first step in the design of practical transport lattices and for use in initializing more detailed beam simulations to evaluate machine performance [2]. The matched envelope solution is typically calculated by numerically integrating trial solutions of the $\mathrm{KV}$ equations from assumed initial condi-

*Electronic address: smlund@llnl.gov
PACS numbers: 29.27.Bd, 41.75.-i, 52.59.Sa, 52.27.Jt

tions over one lattice period and searching for the four initial envelope coordinates and angles that generate the solution with the periodicity of the lattice [2-4]. An elegant formulation of the conventional root finding procedure for envelope matching has been presented by Ryne [4]. Conventional root finding procedures for matching can be surprisingly problematic even for relatively simple focusing lattices. Variations in initial conditions can lead to many inflection points in the envelope functions at the end of the lattice period. Thus initial guesses close to the actual values corresponding to the periodic solution are often necessary to employ standard root finding techniques. This is especially true for complicated focusing lattices with low degrees of symmetry and where the focusing strength (or equivalently, the undepressed single particle phase advance) is large. For large focusing strength and strong space-charge intensity, the matched envelope solution can be unstable over a wide range of system parameters [2,3]. Such instabilities can restrict the basin of attraction when standard numerical root finding methods are used to calculate the needed matching conditions.

In this article we present a new iterative procedure to numerically calculate matched envelope solutions of the $\mathrm{KV}$ equations. The basis of this procedure is the observation that the particle orbits interior to the KV beam must be consistent with the trajectory of the periodic matched beam edge (envelope solution). In the absence of beam space charge, betatron amplitudes calculated from the sinelike and cosinelike principal orbits describing particles moving in the applied focusing fields of the lattice directly specify the matched beam envelope [5]. For finite beam space charge, the principal orbits describing the betatron amplitudes and matched beam envelope cannot be calculated $a$ priori because the defocusing forces from beam space 
charge uniformly distributed within the (undetermined) beam envelope are unknown. In the iterative matching (IM) method, the relations between the betatron amplitudes and the particle orbits are viewed as consistency equations. Starting from a simple trial envelope solution that accounts for both space charge and applied focusing forces in a general manner, the consistency conditions are used to iteratively correct the envelope functions until converged matched envelope solutions are obtained that are consistent with particle orbits internal to the beam.

The IM method offers superior performance and reliability in constructing matched envelopes over conventional root finding because the IM iterations are structured to reflect the periodicity of the actual matched solution rather than searching for parameters that lead to periodicity. The IM method works for all physically achievable system parameters (even in cases of envelope instability) and is most naturally expressed and rapidly convergent when relative beam space-charge strength is expressed in terms of the depressed particle phase advance. All other parametrizations of solutions (specified perveances and emittances, etc.) can also be carried out by simple extensions of the IM method rendering the approach completely general. The natural depressed phase advance parametrization is also useful when carrying out parametric studies because phase advances are the most relevant parameters for analysis of resonancelike effects central to charged particle dynamics in accelerators. The IM method provides a complement to recent analytical perturbation theories developed to construct matched beam envelopes in lattices with certain classes of symmetries [6-9]. In contrast to these analytical theories, the IM method can be applied to arbitrary linear focusing lattices without skew couplings. The highly convergent iterative corrections of the IM method have the same form for all order iterations after seeding, rendering the method straightforward to code and apply to numerically generate accurate matched envelope solutions.

The organization of this paper is the following. After a review of the KV envelope equations in Sec. II, various properties of matched envelope solutions and the continuous focusing limit are analyzed in Sec. III. These results are used in Sec. IV to formulate the IM method for calculation of matched solutions to the $\mathrm{KV}$ envelope equations. Example applications of the IM method are presented in Sec. V to illustrate application and convergence properties of the method over a wide range of system parameters for a variety of systems. Concluding comments in Sec. VI summarize the advantages of the IM method over conventional techniques.

\section{THEORETICAL MODEL}

We consider an unbunched beam of particles of charge $q$ and mass $m$ coasting with axial relativistic factors $\beta_{b}=$ const and $\gamma_{b}=1 / \sqrt{1-\beta_{b}^{2}}$. In the KV model, the beam is propagating in a linear focusing lattice without skew couplings and has uniform charge density within an elliptical cross section with principal radii $r_{x}$ and $r_{y}$ along the transverse $x$ - and $y$-coordinate axes. When self-fields are included and image effects are neglected, the envelope radii consistent with the $\mathrm{KV}$ distribution evolve according to the so-called $\mathrm{KV}$ envelope equations [1-3]

$$
r_{j}^{\prime \prime}(s)+\kappa_{j}(s) r_{j}(s)-\frac{2 Q}{r_{x}(s)+r_{y}(s)}-\frac{\varepsilon_{j}^{2}}{r_{j}^{3}(s)}=0 .
$$

Here, primes denote derivatives with respect to the axial machine coordinate $s$, the subscript $j$ ranges over both transverse coordinates $x$ and $y$, the functions $\kappa_{j}(s)$ represent linear applied focusing forces of the transport lattice, $Q=$ const is the dimensionless perveance, and $\varepsilon_{j}=$ const are the rms edge emittances. Equations relating the functions $\kappa_{j}$ to magnetic and/or electric fields of practical focusing elements are presented in Ref. [3]. The perveance provides a dimensionless measure of self-field defocusing forces internal to the beam [2] and is defined as

$$
Q=\frac{q I}{2 \pi \epsilon_{0} m c^{3} \gamma_{b}^{3} \beta_{b}^{3}} .
$$

Here, $I$ is the constant beam current, $c$ is the speed of light in vacuo, and $\epsilon_{0}$ is the permittivity of free space. The perveance $Q$ can be thought of as a scaled measure of space-charge strength [2]. The rms edge emittances $\varepsilon_{j}$ provide a statistical measure of beam phase-space area projections in $x-x^{\prime}$ and $y-y^{\prime}$ phase-space [2].

When the emittances are constant $\left(\varepsilon_{j}=\right.$ const), the KV envelope equations (1) are consistent with the Vlasov equation only for the KV distribution $[1,10]$, which is a singular function of Courant-Snyder invariants. This singular structure can lead to unphysical instabilities within the Vlasov model [11]. However, the KV envelope equations can be applied to physical (smooth) distributions in an rms equivalent beam sense [2], with the envelope radii and the emittances defined by statistical averages of the physical distribution as

$$
r_{x}=2 \sqrt{\left\langle x^{2}\right\rangle}, \quad r_{y}=2 \sqrt{\left\langle y^{2}\right\rangle}
$$

and

$$
\begin{aligned}
& \varepsilon_{x}=4\left[\left\langle x^{2}\right\rangle\left\langle x^{\prime 2}\right\rangle-\left\langle x x^{\prime}\right\rangle^{2}\right]^{1 / 2}, \\
& \varepsilon_{j}=4\left[\left\langle y^{2}\right\rangle\left\langle y^{\prime 2}\right\rangle-\left\langle y y^{\prime}\right\rangle^{2}\right]^{1 / 2} .
\end{aligned}
$$

Here, $\langle\cdots\rangle$ denotes a transverse statistical average over the beam distribution function. For notational simplicity, we have assumed zero centroid offset (e.g., $\langle x\rangle=0$ ). In this rms equivalent sense, the emittances $\varepsilon_{j}$ will generally evolve in $s$. If this variation has negligible effect on the $r_{j}$, then the KV envelope equations can be applied with $\varepsilon_{j}=$ const to reliably model practical machines. This must 
generally be verified a posteriori with simulations of the full distribution.

For appropriate choices of the lattice focusing functions $\kappa_{j}(s)$, Eq. (1) can be employed to model a wide range of transport channels, including solenoidal and quadrupole transport. For solenoidal transport, the equations must be interpreted in a rotating Larmor frame (see Appendix A of Ref. [3]). In a periodic transport lattice, the $\kappa_{j}$ are periodic with lattice period $L_{p}$, i.e.,

$$
\kappa_{j}\left(s+L_{p}\right)=\kappa_{j}(s) .
$$

The beam envelope is said to be matched to the transport lattice when the envelope functions have the same periodicity as the lattice:

$$
r_{j}\left(s+L_{p}\right)=r_{j}(s) .
$$

For specified focusing functions $\kappa_{j}(s)$, beam perveance $Q$, and emittances $\varepsilon_{j}$, the matching condition is equivalent to requiring that $r_{j}$ and $r_{j}^{\prime}$ satisfy specific initial conditions at $s=s_{i}$ when the envelope equations (1) are integrated as an initial value problem. The required initial conditions generally vary with the phase of $s_{i}$ in the lattice period (because the conditions vary with the local matched solution). In conventional procedures for envelope matching, needed initial conditions are typically found by numerical root finding starting from guessed seed values [3]. This numerical matching can be especially problematic when: applied focusing strengths are large, the focusing lattice is complicated and devoid of symmetries that can reduce the dimensionality of the root finding, choices of solution parameters require extra constraints to effect, and where the matched beam envelope is unstable.

The undepressed particle phase advance per lattice period $\sigma_{0 j}$ provides a dimensionless measure of the strength of the applied focusing functions $\kappa_{j}$ describing the periodic lattice [3,5]. The $\sigma_{0 j}$ can be calculated from [5]

$$
\cos \sigma_{0 j}=\frac{1}{2} \operatorname{Tr} \mathbf{M}_{0 j}\left(s_{i}+L_{p} \mid s_{i}\right),
$$

where $\mathbf{M}_{0 j}\left(s \mid s_{i}\right)$ denotes the $2 \times 2$ single particle transfer matrix in the $j$-plane from axial coordinate $s_{i}$ to $s$. Explicitly, we have

$$
\mathbf{M}_{0 j}\left(s \mid s_{i}\right)=\left(\begin{array}{ll}
C_{0 j}\left(s \mid s_{i}\right) & S_{0 j}\left(s \mid s_{i}\right) \\
C_{0 j}^{\prime}\left(s \mid s_{i}\right) & S_{0 j}^{\prime}\left(s \mid s_{i}\right)
\end{array}\right),
$$

where the $C_{0 j}\left(s \mid s_{i}\right)$ and $S_{0 j}\left(s \mid s_{i}\right)$ denote cosinelike and sinelike principal orbit functions satisfying

$$
F_{0 j}^{\prime \prime}\left(s \mid s_{i}\right)+\kappa_{j}(s) F_{0 j}\left(s \mid s_{i}\right)=0,
$$

with $F$ representing $C$ or $S$ with $C_{0 j}$ subject to cosinelike initial $\left(s=s_{i}\right)$ conditions $C_{0 j}\left(s_{i} \mid s_{i}\right)=1$ and $C_{0 j}^{\prime}\left(s_{i} \mid s_{i}\right)=$ 0 , and with $S_{0 j}$ subject to sinelike initial conditions $S_{0 j}\left(s_{i} \mid s_{i}\right)=0$ and $S_{0 j}^{\prime}\left(s_{i} \mid s_{i}\right)=1$. Equation (7) can be expressed in terms of $C_{0 j}$ and $S_{0 j}^{\prime}$ as

$$
\cos \sigma_{0 j}=\frac{1}{2}\left[C_{0 j}\left(s_{i}+L_{p} \mid s_{i}\right)+S_{0 j}^{\prime}\left(s_{i}+L_{p} \mid s_{i}\right)\right] .
$$

The $\sigma_{0 j}$ are independent of the particular value of $s_{i}$ used in the calculation of the principal functions. For some particular cases such as piecewise constant $\kappa_{j}$, the principal functions $F_{0 j}$ can be calculated analytically. But, in general, the $F_{0 j}$ must be calculated numerically. In the absence of space charge, the particle orbit is stable whenever $\sigma_{0 j}<180^{\circ}$ and parametric bands of stability can also usually be found for $\sigma_{0 j}>180^{\circ}$ [2,3,12]. For a stable orbit, the scale of the $\kappa_{j}$ (i.e., $\kappa_{j} \rightarrow \alpha \kappa_{j}$ with $\alpha=$ const setting the scale of the specified $\kappa_{j}$ ) can always be regarded as being set by the $\sigma_{0 j}$. In this context, Eq. (10) is employed to fix the scale of the $\kappa_{j}$ in terms of $\sigma_{0 j}$ and other parameters defining the $\kappa_{j}$. Because there appears to be no advantage in using stronger focusing with $\sigma_{0 j}>180^{\circ}$ in terms of producing more radially compact matched envelopes $[3,13]$, we will assume in all analysis that follows that the $\kappa_{j}$ are sufficiently weak to satisfy $\sigma_{0}<180^{\circ}$.

The formulation given above for calculation of the undepressed principal orbits $C_{0 j}$ and $S_{0 j}$ and the undepressed particle phase advances $\sigma_{0 j}$ can also be applied to calculate the depressed principal orbits $C_{j}$ and $S_{j}$ and the depressed phase advances $\sigma_{j}$ in the presence of uniform beam spacecharge density for a particle moving within the matched $\mathrm{KV}$ beam envelopes. This is done by replacing

$$
\kappa_{j} \rightarrow \kappa_{j}-\frac{2 Q}{\left(r_{x}+r_{y}\right) r_{j}}
$$

in Eq. (9) and dropping the subscript 0s in Eqs. (7)-(10) for notational clarity (i.e., $C_{0 j} \rightarrow C_{j}$ and $S_{0 j} \rightarrow S_{j}$ ). Explicitly, the depressed principal functions satisfy

$$
F_{j}^{\prime \prime}\left(s \mid s_{i}\right)+\kappa_{j}(s) F_{j}\left(s \mid s_{i}\right)-\frac{2 Q F_{j}\left(s \mid s_{i}\right)}{\left[r_{x}(s)+r_{y}(s)\right] r_{j}(s)}=0,
$$

with $F$ representing $C$ or $S$ with $C_{j}$ subject to $C_{j}\left(s_{i} \mid s_{i}\right)=1$ and $C_{j}^{\prime}\left(s_{i} \mid s_{i}\right)=0$, and $S_{j}$ subject to $S_{j}\left(s_{i} \mid s_{i}\right)=0$ and $S_{j}^{\prime}\left(s_{i} \mid s_{i}\right)=1$, and the depressed phase advances satisfy

$$
\cos \sigma_{j}=\frac{1}{2}\left[C_{j}\left(s_{i}+L_{p} \mid s_{i}\right)+S_{j}^{\prime}\left(s_{i}+L_{p} \mid s_{i}\right)\right] .
$$

For a stable orbit, it can be shown that the $\sigma_{j}$ can also be calculated from the matched envelope as $[3,5]$

$$
\sigma_{j}=\varepsilon_{j} \int_{s_{i}}^{s_{i}+L_{p}} \frac{d s}{r_{j}^{2}(s)} .
$$

This formula can also be applied to calculate $\sigma_{0 j}$ by using the matched envelope functions $r_{j}$ calculated with $Q=0$.

Matched envelope solutions of Eq. (1) can be regarded as being determined by the focusing functions $\kappa_{j}$, the perveance $Q$, and the emittances $\varepsilon_{j}$. The lattice period $L_{p}$ is implicitly specified through the $\kappa_{j}$. We will always 
TABLE I. Possible parametrizations of matched envelope solutions.

\begin{tabular}{ll}
\hline \hline Case & \multicolumn{1}{c}{ Parameters } \\
\hline 0 & $\kappa_{j}\left(\sigma_{0 j}\right), Q, \varepsilon_{j}$ \\
1 & $\kappa_{j}\left(\sigma_{0 j}\right), Q, \sigma_{j}$ \\
2 & $\kappa_{j}\left(\sigma_{0 j}\right), \varepsilon_{j}$, and one of $\sigma_{j}$ \\
3 & $\kappa_{j}\left(\sigma_{0 j}\right), \sigma_{j}$, and one of $\varepsilon_{j}$ \\
\hline \hline
\end{tabular}

regard the scale of the $\kappa_{j}$ as being set by the undepressed phase advances $\sigma_{0 j}$ through Eq. (10). For $\sigma_{0 j}<180^{\circ}$ there is no ambiguity in scale choice and the use of the $\sigma_{0 j}$ as parameters enables disparate classes of lattices to be compared in a common framework [3]. The depressed phase advances $\sigma_{x}$ and $\sigma_{y}$ can be employed to replace up to two of the three parameters $Q, \varepsilon_{x}$, and $\varepsilon_{y}$. Such replacements can be convenient, particularly when carrying out parametric surveys (for example, see Ref. [3]) because $\sigma_{j} / \sigma_{0 j}$ is a dimensionless measure of space-charge strength satisfying $0 \leq \sigma_{j} / \sigma_{0 j} \leq 1$ with $\sigma_{j} / \sigma_{0 j} \rightarrow 1$ representing a warm beam with negligible space charge (i.e., $Q \rightarrow 0$, or $\varepsilon_{j} \rightarrow \infty$ for finite $Q$ ), and $\sigma_{j} / \sigma_{0 j} \rightarrow 0$ representing a cold beam with maximum space-charge intensity (i.e., $\varepsilon_{j} \rightarrow$ $\infty)$. We will discuss calculation of matched beam envelopes for the useful parametrization cases listed in Table I. In cases typical of linear accelerators the focusing functions have equal strength in the $x$ - and $y$-planes giving $\sigma_{0 x}=\sigma_{0 y}$. In such plane-symmetric cases we denote $\sigma_{0 j} \equiv \sigma_{0}$. In practical situations where the focusing lattice and emittances are both plane symmetric with $\sigma_{0 j} \equiv \sigma_{0}$ and $\varepsilon_{j} \equiv \varepsilon$, then the depressed phase advance is also plane symmetric with $\sigma_{j} \equiv \sigma$ and parametrization cases 2 and 3 are identical. It is assumed that a unique matched envelope solution exists independent of the parametrization when the $\kappa_{j}$ are fully specified. There is no known proof of this conjecture, but numerical evidence suggests that it is correct for simple focusing lattices (i.e., simple $\kappa_{j}$ ) when $\sigma_{0 j}<180^{\circ}$. In typical experimental situations, note that transport lattices are fixed in geometry and excitations of focusing elements in the lattices can be individually adjusted. In the language adopted here, such lattices with different excitations in focusing elements (both overall scale and otherwise) correspond to different lattices described by different $\kappa_{j}$ with different matched envelopes.

\section{MATCHED ENVELOPE PROPERTIES}

In development of the IM method in Sec. IV, we employ a consistency equation between depressed particle orbits within the beam and the matched envelope functions (III A) and use a continuous focusing description of the matched beam (IIIB) to model space-charge forces in construction of a seed iteration. Henceforth, we denote lattice period averages with overbars, i.e., for some quantity $\zeta(s)$,

$$
\bar{\zeta} \equiv \int_{s_{i}}^{s_{i}+L_{p}} \frac{d s}{L_{p}} \zeta(s)
$$

\section{A. Consistency condition between particle orbits and the matched envelope}

We calculate nonlinear consistency conditions for the matched envelope functions $r_{j}$ and the depressed principal orbit functions $C_{j}$ and $S_{j}$ as follows. First, the transfer matrix $\mathbf{M}_{j}$ of the depressed particle orbit in the $j$-plane is expressed in terms of betatron function like formulation as [5]

$$
\mathbf{M}_{j}\left(s \mid s_{i}\right)=\left(\begin{array}{ll}
C_{j}\left(s \mid s_{i}\right) & S_{j}\left(s \mid s_{i}\right) \\
C_{j}^{\prime}\left(s \mid s_{i}\right) & S_{j}^{\prime}\left(s \mid s_{i}\right)
\end{array}\right)
$$

with

$$
\begin{aligned}
C_{j}\left(s \mid s_{i}\right)= & \frac{r_{j}(s)}{r_{j}\left(s_{i}\right)} \cos \Delta \psi_{j}(s)-\frac{r_{j}^{\prime}\left(s_{i}\right) r_{j}(s)}{\varepsilon_{j}} \sin \Delta \psi_{j}(s), \\
S_{j}\left(s \mid s_{i}\right)= & \frac{r_{j}\left(s_{i}\right) r_{j}(s)}{\varepsilon_{j}} \sin \Delta \psi_{j}(s), \\
C_{j}^{\prime}\left(s \mid s_{i}\right)= & {\left[\frac{r_{j}^{\prime}(s)}{r_{j}\left(s_{i}\right)}-\frac{r_{j}^{\prime}\left(s_{i}\right)}{r_{j}(s)}\right] \cos \Delta \psi_{j}(s) } \\
& -\left[\frac{\varepsilon_{j}}{r_{j}\left(s_{i}\right) r_{j}(s)}+\frac{r_{j}^{\prime}\left(s_{i}\right) r_{j}^{\prime}(s)}{\varepsilon_{j}}\right] \sin \Delta \psi_{j}(s), \\
S_{j}^{\prime}\left(s \mid s_{i}\right)= & \frac{r_{j}\left(s_{i}\right)}{r_{j}(s)} \cos \Delta \psi_{j}(s)+\frac{r_{j}\left(s_{i}\right) r_{j}^{\prime}(s)}{\varepsilon_{j}} \sin \Delta \psi_{j}(s) .
\end{aligned}
$$

Here,

$$
\Delta \psi_{j}(s)=\varepsilon_{j} \int_{s_{i}}^{s} \frac{d \tilde{s}}{r_{j}^{2}(\tilde{s})}
$$

is the change in betatron phase of the particle orbit from $s=s_{i}$ to $s$ and the principal functions $C_{j}$ and $S_{j}$ are calculated including the linear space-charge term of the uniform density elliptical beam from Eq. (12). Note that $r_{j} \equiv \sqrt{\varepsilon_{j} \beta_{j}}$ can be used in Eqs. (17) and (18) to express the results more conventionally in terms of the betatron amplitude functions $\beta_{j}$ describing linear orbits internal to the beam in the $j$-plane [5]. These generalized betatron functions are periodic [i.e., $\beta_{j}\left(s+L_{p}\right)=\beta_{j}(s)$ ] and include the transverse defocusing effects of uniformly distributed space charge within the $\mathrm{KV}$ equilibrium envelope. Recognizing that $\Delta \psi_{j}\left(s_{i}+L_{p}\right)=\sigma_{j}$ [see Eq. (14)] and that the matched envelope functions $r_{j}$ have period $L_{p}$ gives 


$$
\beta_{j}(s)=\frac{r_{j}^{2}(s)}{\varepsilon_{j}}=\frac{\left[\mathbf{M}_{j}\right]_{12}\left(s+L_{p} \mid s\right)}{\sin \sigma_{j}}=\frac{S_{j}\left(s+L_{p} \mid s\right)}{\sin \sigma_{j}} .
$$

Here, $\left[\mathbf{M}_{j}\right]_{12}$ denotes the 1,2 component of the $2 \times 2$ matrix $\mathbf{M}_{j}$ and $\sigma_{j}$ can be equivalently calculated from either Eq. (13) or Eq. (14).

Equation (19) can be applied to numerically calculate the consistency conditions for the matched envelope functions $r_{j}$ on a discretized axial grid of $s$ locations. As written, the principal orbit functions employed (i.e., the $C_{j}$ and $S_{j}$ ) need to be independently calculated at each $s$-location on the grid through one lattice period. The fact that every period is the same can be applied to simplify the calculation. For any initial axial coordinate $s_{i} \quad$ we have $\mathbf{M}_{j}\left(s+L_{p} \mid s\right)=\mathbf{M}_{j}\left(s+L_{p} \mid s_{i}+L_{p}\right) \cdot$ $\mathbf{M}_{j}\left(s_{i}+L_{p} \mid s\right)$. Multiplying this equation from the right side by the identity matrix $\mathbf{I}=\mathbf{M}_{j}\left(s \mid s_{i}\right) \cdot \mathbf{M}_{j}^{-1}\left(s \mid s_{i}\right)$ where $\mathbf{M}_{j}^{-1}$ is the inverse matrix and using $\mathbf{M}_{j}\left(s_{i}+L_{p} \mid s\right)$. $\mathbf{M}_{j}\left(s \mid s_{i}\right)=\mathbf{M}_{j}\left(s_{i}+L_{p} \mid s_{i}\right)$ gives

$$
\mathbf{M}_{j}\left(s+L_{p} \mid s\right)=\mathbf{M}_{j}\left(s \mid s_{i}\right) \cdot \mathbf{M}_{j}\left(s_{i}+L_{p} \mid s_{i}\right) \cdot \mathbf{M}_{j}^{-1}\left(s \mid s_{i}\right) .
$$

Some straightforward algebra employing Eqs. (16), (19), and (20), and the Wronskian (or symplectic) condition on $\mathbf{M}_{j}[5]$

$$
C_{j}\left(s \mid s_{i}\right) S_{j}^{\prime}\left(s \mid s_{i}\right)-S_{j}\left(s \mid s_{i}\right) C_{j}^{\prime}\left(s \mid s_{i}\right)=1
$$

yields

$$
\begin{aligned}
\beta_{j}(s)= & \frac{r_{j}^{2}(s)}{\varepsilon_{j}} \\
= & \frac{S_{j}^{2}\left(s \mid s_{i}\right)}{S_{j}\left(s_{i}+L_{p} \mid s_{i}\right) / \sin \sigma_{j}}+\frac{S_{j}\left(s_{i}+L_{p} \mid s_{i}\right)}{\sin \sigma_{j}} \\
& \times\left[C_{j}\left(s \mid s_{i}\right)+\frac{\cos \sigma_{j}-C_{j}\left(s_{i}+L_{p} \mid s_{i}\right)}{S_{j}\left(s_{i}+L_{p} \mid s_{i}\right)} S_{j}\left(s \mid s_{i}\right)\right]^{2} .
\end{aligned}
$$

Equation (22) explicitly shows that the linear principal functions $C_{j}$ and $S_{j}$ need only be calculated in $s$ from some arbitrary initial point $\left(s_{i}\right)$ over one lattice period (to $s_{i}+L_{p}$ ) to calculate the consistency condition for the matched envelope functions $r_{j}(s)$, or equivalently, the betatron amplitude functions $\beta_{j}(s) \equiv r_{j}^{2}(s) / \varepsilon_{j}$. Equation (22) can also be derived using Courant-Snyder invariants of particle orbits within the beam.

Equations (13) and (22) form the foundation of an iterative numerical method developed in Sec. IV to calculate the matched beam envelope for any lattice. These equations express the intricate connection between the bundle of depressed particle orbits within the uniform density $\mathrm{KV}$ beam and the locus of maximum particle excursions defining the envelope functions $r_{j}$. The method will be iterative because the consistent matched envelope functions $r_{j}$ are necessary to integrate the linear differential equations for the depressed orbit principal functions $C_{j}$ and $S_{j}$. However, in the limit $Q \rightarrow 0$, the principal functions do not depend on the $r_{j}$ and the matched envelope can be immediately calculated from the equations. Thus, the periodic zero-current matched beam envelope can be directly calculated using Eq. (22) in terms of the two independent, aperiodic linear orbits (i.e., $C_{0 j}$ and $S_{0 j}$ ) integrated over one lattice period.

Additional constraints on the matched envelope functions $r_{j}$ and/or betatron functions $\beta_{j}$ are necessary to formulate the IM method for parametrizations where one or more of the parameters $Q$ and $\varepsilon_{j}$ need to be eliminated (see Table I). Appropriate constraints can be derived by taking the period average of Eq. (1) for a matched envelope, giving

$$
\overline{\kappa_{j} r_{j}}-2 Q \overline{\frac{1}{r_{x}+r_{y}}}-\varepsilon_{j}^{2} \frac{\overline{1}}{r_{j}^{3}}=0 .
$$

\section{B. Continuous limit}

In the continuous focusing approximation, we take the lattice focusing functions $\kappa_{j}$ as constants set according to

$$
\kappa_{j} \rightarrow\left(\frac{\sigma_{0 j}}{L_{p}}\right)^{2}
$$

with the $\sigma_{0 j}$ calculated from Eq. (10) consistent with the actual $s$-varying periodic focusing functions $\kappa_{j}$. Then we replace $r_{j} \rightarrow \overline{r_{j}}$ in the KV envelope equations (1) and take $\overline{r_{j}}=$ const to obtain the continuous limit envelope equation

$$
\left(\frac{\sigma_{0 j}}{L_{p}}\right)^{2} \overline{r_{j}}-\frac{2 Q}{\overline{r_{x}}+\overline{r_{y}}}-\frac{\varepsilon_{j}^{2}}{{\overline{r_{j}}}^{3}}=0
$$

Equation (25) provides an estimate of the lattice period average envelope radii $\overline{r_{j}}$ in response to the applied focusing and defocusing forces from beam space-charge and thermal (emittance) effects. Solutions for $\overline{r_{j}}$ will be employed to seed the IM method of constructing matched envelope solutions. In general, the continuous limit approximations tend to be more accurate for weaker applied focusing strengths with $\sigma_{0 j} \lesssim 80^{\circ}$. However, even for higher values of $\sigma_{0 j}<180^{\circ}$, the formulas can still be applied to seed iterative numerical matching methods if the methods have a sufficiently large "basin of attraction" to the desired solution.

For case 0 parametrizations (specified $\sigma_{0 j}, Q$, and $\varepsilon_{j}$ ), the solutions of Eq. (25) will, in general, need to be calculated numerically from a trial guess. Certain limits are analytically accessible and often relevant. If the beam perveance $Q$ is zero, or equivalently if $\sigma_{j}=\sigma_{0 j}$, then the solutions of Eq. (25) are decoupled and are trivially ex- 
pressed as

$$
\overline{r_{j}}=\sqrt{\frac{\varepsilon_{j}}{\left(\sigma_{0 j} / L_{p}\right)}} .
$$

Alternatively, this result can be obtained using $r_{j}=\overline{r_{j}}$ in Eq. (14) with $\sigma_{j} \rightarrow \sigma_{0 j}$. In the case of a symmetric system with $\sigma_{0 x}=\sigma_{0 y} \equiv \sigma_{0}$ and $\varepsilon_{x}=\varepsilon_{y} \equiv \varepsilon$, then $\overline{r_{x}}=\overline{r_{y}} \equiv$ $\overline{r_{b}}$ and the solutions of Eq. (25) decouple and the resulting quadratic equation in ${\overline{r_{b}}}^{2}$ is solved as

$$
\overline{r_{b}}=\frac{1}{\left(\sigma_{0} / L_{p}\right)}\left[\frac{Q}{2}+\frac{1}{2} \sqrt{Q^{2}+4\left(\frac{\sigma_{0}}{L_{p}}\right)^{2} \varepsilon^{2}}\right]^{1 / 2} .
$$

In parametrization cases $1-3$, the continuous limit solutions $\overline{r_{j}}$ must be expressed using the depressed phase advances $\sigma_{j}$ to eliminate one or more of the parameters $Q$ and $\varepsilon_{j}$. In these cases, if the emittances $\varepsilon_{j}$ are known, then Eq. (14) can be employed to estimate

$$
\overline{r_{j}}=\sqrt{\frac{\varepsilon_{j}}{\left(\sigma_{j} / L_{p}\right)}} .
$$

Alternatively, if the perveance $Q$ is known but one or more of the emittances $\varepsilon_{j}$ is unknown, we can use Eq. (28) to eliminate the emittance term(s) in Eq. (25) obtaining $\left(\sigma_{0 j}^{2}-\sigma_{j}^{2}\right) \overline{r_{j}}=2 Q L_{p}^{2} /\left(\overline{r_{x}}+\overline{r_{y}}\right)$. Taking the ratio of the $x$ - and $y$-equations yields

$$
\frac{\overline{r_{y}}}{\overline{r_{x}}}=\frac{\sigma_{0 x}^{2}-\sigma_{x}^{2}}{\sigma_{0 y}^{2}-\sigma_{y}^{2}} .
$$

Back-substitution of this result in $\left(\sigma_{0 j}^{2}-\sigma_{j}^{2}\right) \overline{r_{j}}=$ $2 Q L_{p}^{2} /\left(\overline{r_{x}}+\overline{r_{y}}\right)$ then gives

$$
\begin{gathered}
\bar{r}_{x}=\frac{\sqrt{2 Q} L_{p}}{\sqrt{\left(\sigma_{0 x}^{2}-\sigma_{x}^{2}\right)+\frac{\left(\sigma_{0 x}^{2}-\sigma_{x}^{2}\right)^{2}}{\left(\sigma_{0 y}^{2}-\sigma_{y}^{2}\right)}}} \\
\bar{r}_{y}=\frac{\sqrt{2 Q} L_{p}}{\sqrt{\left(\sigma_{0 y}^{2}-\sigma_{y}^{2}\right)+\frac{\left(\sigma_{0 y}^{2}-\sigma_{y}^{2}\right)^{2}}{\left(\sigma_{0 x}^{2}-\sigma_{x}^{2}\right)}}} .
\end{gathered}
$$

Smooth-limit formulations in Refs. [14,15] can also be employed to estimate the $\overline{r_{j}}$ for systems with high degrees of symmetry.

\section{NUMERICAL ITERATIVE METHOD FOR MATCHED ENVELOPE CALCULATION}

We formulate a numerical iterative matching (IM) method to construct the matched beam envelope functions $r_{j}(s)$ over one lattice period $L_{p}$ using the developments in Sec. III. The IM method is formulated for arbitrary periodic focusing functions $\kappa_{j}$. Constraints necessary to apply the IM formalism to all cases of envelope parametrizations listed in Table I are derived.
Label all quantities varying with the iteration number with a superscript $i(i=0,1,2, \ldots)$ denoting the iteration order. For example, the $i$ th order envelope functions are labeled $r_{j}^{i}$. The iteration label should not be confused with the initial coordinate $s_{i}$ and the initial "seed" iteration corresponds to $i=0$. Parameters such as the perveance $Q$ or emittances $\varepsilon_{j}$ will also be superscripted to cover parametrization cases where the quantities are unspecified and are calculated from the envelope functions and other parameters (see Table I). For example, $\varepsilon_{j}^{i}$ denotes the $j$-plane emittance at the $i$ th iteration. For parametrization cases where the value of $\varepsilon_{j}$ is specified, then $\varepsilon_{j}^{i}=\varepsilon_{j} \equiv$ const.

For iterations $i \geq 1$, we calculate refinements of the principal orbit functions [see Eq. (12)] in terms of the envelope calculated at the previous, $i-1$ iteration from

$$
F_{j}^{i \prime \prime}+\kappa_{j} F_{j}^{i}-\frac{2 Q^{i-1} F_{j}^{i}}{\left(r_{x}^{i-1}+r_{y}^{i-1}\right) r_{j}^{i-1}}=0 .
$$

Here, $F_{j}^{i}$ denotes $C_{j}^{i}\left(s \mid s_{i}\right)$ or $S_{j}^{i}\left(s \mid s_{i}\right)$ which are subject to the initial $\left(s=s_{i}\right)$ conditions $C_{j}^{i}\left(s_{i} \mid s_{i}\right)=1, C_{j}^{i \prime}\left(s_{i} \mid s_{i}\right)=0$ and $S_{j}^{i}\left(s_{i} \mid s_{i}\right)=0, S_{j}^{i \prime}\left(s_{i} \mid s_{i}\right)=1$. Note that the $F_{j}^{i}$ depend on the envelope functions $r_{j}^{i-1}$ and perveance $Q^{i-1}$ of the prior, $i-1$, iteration. Updated envelope functions $r_{j}^{i}$ and/ or betatron functions $\beta_{j}^{i}$ are calculated [see Eq. (22)] from the $F_{j}^{i}$ for all $i$ from

$$
\begin{aligned}
\beta_{j}^{i}(s)= & \frac{\left[r_{j}^{i}(s)\right]^{2}}{\varepsilon_{j}^{i}} \\
= & \frac{\left[S_{j}^{i}\left(s \mid s_{i}\right)\right]^{2}}{S_{j}^{i}\left(s_{i}+L_{p} \mid s_{i}\right) / \sin \sigma_{j}^{i}}+\frac{S_{j}^{i}\left(s_{i}+L_{p} \mid s_{i}\right)}{\sin \sigma_{j}^{i}} \\
& \times\left[C_{j}^{i}\left(s \mid s_{i}\right)+\frac{\cos \sigma_{j}^{i}-C_{j}^{i}\left(s_{i}+L_{p} \mid s_{i}\right)}{S_{j}^{i}\left(s_{i}+L_{p} \mid s_{i}\right)} S_{j}^{i}\left(s \mid s_{i}\right)\right]^{2} .
\end{aligned}
$$

Here, if the parametrization does not specify the depressed phase advances as $\sigma_{j}^{i}=\sigma_{j}$, then they are calculated [see Eq. (13)] for all $i$ from

$$
\cos \sigma_{j}^{i}=\frac{1}{2}\left[C_{j}^{i}\left(s_{i}+L_{p} \mid s_{i}\right)+S_{j}^{i \prime}\left(s_{i}+L_{p} \mid s_{i}\right)\right] .
$$

In parametrization cases 0 to 3 (see Table I), one or more of the needed quantities among $Q^{i}, \varepsilon_{j}^{i}$, and $\sigma_{j}^{i}$ are not specified (e.g., for case $1 \varepsilon_{j}^{i}$ is undetermined: $\varepsilon_{j}^{i} \neq \varepsilon_{j}$ ) and must be calculated to apply Eq. (32) and/or to calculate the next $(i+1)$ iteration principal functions from Eq. (31). Equations (33) and/or the constraint equations (23) with $Q \rightarrow Q^{i}, \varepsilon_{j} \rightarrow \varepsilon_{j}^{i}$, and $r_{j} \rightarrow r_{j}^{i}$ (or in some cases $r_{j} \rightarrow$ $\sqrt{\varepsilon_{j}^{i} \beta_{j}^{i}}$ ) can be employed to calculate parameter eliminations necessary to fully realize each iteration for each case as follows:

Case $0\left(\kappa_{j}, Q, \varepsilon_{j}\right.$ specified). The $\sigma_{j}^{i}$ can be calculated from Eq. (33). 
Case $1\left(\kappa_{j}, Q\right.$, and $\sigma_{j}$ specified). The $\varepsilon_{j}^{i}$ can be calculated using Eq. (23) expressed in betatron form to obtain

$$
\begin{aligned}
\frac{\varepsilon_{x}^{i}}{2 Q^{i}} & =\frac{\frac{1}{\sqrt{\beta_{x}^{i}}+\sqrt{\varepsilon_{y}^{i} / \varepsilon_{x}^{i}} \sqrt{\beta_{y}^{i}}}}{\kappa_{x} \sqrt{\beta_{x}^{i}}-\overline{1 /\left(\beta_{x}^{i}\right)^{3 / 2}}}, \\
\frac{\varepsilon_{y}^{i}}{2 Q^{i}} & =\frac{\frac{1}{\sqrt{\varepsilon_{x}^{i} / \varepsilon_{y}^{i}} \sqrt{\beta_{x}^{i}}+\sqrt{\beta_{y}^{i}}}}{\kappa_{y} \sqrt{\beta_{y}^{i}}-\overline{1 /\left(\beta_{y}^{i}\right)^{3 / 2}}},
\end{aligned}
$$

with the ratio $\varepsilon_{y}^{i} / \varepsilon_{x}^{i}$ on the right-hand side of the equations determined by

$$
\sqrt{\frac{\varepsilon_{y}^{i}}{\varepsilon_{x}^{i}}}=\frac{\overline{\kappa_{x} \sqrt{\beta_{x}^{i}}}-\overline{1 /\left(\beta_{x}^{i}\right)^{3 / 2}}}{\kappa_{y} \sqrt{\beta_{y}^{i}}}-\overline{1 /\left(\beta_{y}^{i}\right)^{3 / 2}} .
$$

Note that expressing the constraints in terms of betatron functions $\beta_{j}^{i}$ is necessary in this case because the envelope functions $r_{j}^{i}$ cannot be calculated from Eq. (32) until the $\varepsilon_{j}^{i}$ are known, whereas, because of the structure of the envelope equations, the $\beta_{j}^{i}=\left(r_{j}^{i}\right)^{2} / \varepsilon_{j}^{i}$ can be calculated from Eq. (32) without a priori knowledge of the values of $\varepsilon_{j}^{i}$.

Case $2\left(\kappa_{j}, \varepsilon_{j}\right.$, and $\sigma_{x}$ specified; or $\kappa_{j}, \varepsilon_{j}$, and $\sigma_{y}$ specified). If necessary, either $\sigma_{x}^{i}$ or $\sigma_{y}^{i}$ can be calculated from Eq. (33) to enable full specification of the functions $\beta_{j}^{i}$ or $r_{j}^{i}$. Then, $Q^{i}$ can be calculated using Eq. (34) and the $\beta_{j}^{i}$, or alternatively, using

$$
2 Q^{i}=\frac{\overline{\kappa_{j} r_{j}^{i}}-\overline{\left(\varepsilon_{j}^{i}\right)^{2} /\left(r_{j}^{i}\right)^{3}}}{\overline{1 /\left(r_{x}^{i}+r_{y}^{i}\right)}}
$$

with $\varepsilon_{j}^{i}=\varepsilon_{j}$.

Case $3\left(\kappa_{j}, \sigma_{j}\right.$, and $\varepsilon_{x}$ specified; or $\kappa_{j}, \sigma_{j}$, and $\varepsilon_{y}$ specified). First, Eq. (35) and the $\beta_{j}^{i}$ functions can be applied to calculate $\varepsilon_{y}^{i}$ from specified $\varepsilon_{x}$, or $\varepsilon_{x}^{i}$ from specified $\varepsilon_{y}$. Then, $Q^{i}$ can be calculated from the $\varepsilon_{j}^{i}$ (if specified, $\varepsilon_{j}^{i}=\varepsilon_{j}$ ) using Eq. (34) and the $\beta_{j}^{i}$, or alternatively, with Eq. (36) and the $r_{j}^{i}$.

The seed $i=0$ iteration is treated as a special case where the continuous limit formulas derived in Sec. III B are applied to estimate the leading-order defocusing effect of space charge on the beam. In this case the principal functions are calculated from

$$
F_{j}^{0 \prime \prime}+\kappa_{j} F_{j}^{0}-\frac{2 \bar{Q} F_{j}^{0}}{\left(\overline{r_{x}}+\overline{r_{y}}\right) \overline{r_{j}}}=0 .
$$

Here, $F_{j}^{0}$ denotes $C_{j}^{0}\left(s \mid s_{i}\right)$ or $S_{j}^{0}\left(s \mid s_{i}\right)$ subject to the initial $\left(s=s_{i}\right) \quad$ conditions $C_{j}^{0}\left(s_{i} \mid s_{i}\right)=1, \quad C_{j}^{0 \prime}\left(s_{i} \mid s_{i}\right)=0 \quad$ and $S_{j}^{0}\left(s_{i} \mid s_{i}\right)=0, S_{j}^{0 \prime}\left(s_{i} \mid s_{i}\right)=1$, and $\bar{Q}$ and $\overline{r_{j}}$ denote the continuous focusing approximation perveance and envelopes calculated from the formulation in Sec. III B with $Q \rightarrow \bar{Q}$ and $\varepsilon_{j} \rightarrow \overline{\varepsilon_{j}}$. The continuous focusing values of $\bar{Q}$ and $\overline{\varepsilon_{j}}$ used in calculating the $\overline{r_{j}}$ are set by the parametrization values in cases where they are specified (e.g., $\bar{Q}=Q$ for $Q$ specified). Otherwise, $\bar{Q}$ and/or the $\overline{\varepsilon_{j}}$ are calculated in terms of other parameters using the appropriate constraint equations from Eqs. (26)-(30) applied with $Q \rightarrow \bar{Q}$ and $\varepsilon_{j} \rightarrow \overline{\varepsilon_{j}}$.

Note that the seed envelope functions $r_{j}^{0}$ calculated under this procedure are not the continuous limit functions (i.e., $r_{j}^{0} \neq \overline{r_{j}}$ ). Likewise, in parametrizations where they are not held fixed, the seed perveance and emittances will not equal the continuous focusing values (i.e., $Q^{0} \neq \bar{Q}$ and/or $\varepsilon_{j}^{0} \neq \overline{\varepsilon_{j}}$ ). Because of Eq. (32), the seed envelope functions $r_{j}^{0}$ will have a (dominant) contribution to the envelope flutter from the applied focusing fields of the lattice with a correction due to space-charge defocusing forces derived from the continuous limit formulas. This approximation should produce seed envelope functions $r_{j}^{0}$ that are significantly closer to the actual periodic envelope functions $r_{j}$ than would be obtained by simply applying continuous limit formulas (i.e., taking $r_{j}^{0}=\overline{r_{j}}$ ) or by neglecting the effects of space charge altogether [i.e., by calculating $r_{j}^{0}$ using Eq. (22) with $Q=0$ ]. Generally speaking, a seed iteration closer to the desired solution can reduce the number of iterations required to achieve tolerance, and more importantly, can help ensure a starting point within the basin of attraction of the method, thereby reducing the likelihood of algorithm failure. At the expense of greater complexity and less lattice generality, alternative seed iterations can be generated using low-order terms from analytical perturbation theories for matched envelope solutions [6-9]. In certain cases, these formulations may generate seed iterations closer to the matched solution.

Iterations can be terminated at some value of $i$ where the maximum fractional change between the $i$ and $(i-1)$ iterations is less than a specified tolerance tol, i.e.,

$$
\operatorname{Max}\left|\frac{r_{j}^{i}-r_{j}^{i-1}}{r_{j}^{i}}\right| \leq \text { tol, }
$$

where Max denotes the maximum taken over the lattice period $L_{p}$ and the component index $j=x, y$. Many numerical methods will be adequate for solving the linear ordinary differential equations for the principal functions $C_{j}^{i}$ and $S_{j}^{i}$ of the iteration because they are only required over one lattice period. Generally, the principal functions will be solved at (uniformly spaced) discrete points in $s$ over the lattice period. These discretized solutions can then be employed with quadrature formulas to calculate any needed integral constraints to affect the envelope parametrizations given in Table I. Finally, the convergence criterion (38) can be evaluated at the discrete $s$-values of the numerical solution for $r_{j}^{i}$ at the $i$ th iteration using saved previous iteration values for $r_{j}^{i-1}$ that are needed for calculation of the $i$ th iteration. It is usually sufficient to 
evaluate the convergence criterion at some limited, randomly distributed sample of $s$-values within the lattice period. Issues of convergence rate and the basin of attraction of the method are parametrically analyzed for examples corresponding to typical classes of transport lattices in Sec. V.

\section{EXAMPLE APPLICATIONS}

In this section we present examples of the IM method developed in Sec. IV to construct matched envelope solutions and explore parametric convergence properties for example solenoidal and quadrupole periodic focusing lattices. For simplicity, examples are restricted to planesymmetric focusing lattices with equal undepressed particle phase advances in the $x$ - and $y$-planes (i.e., $\sigma_{0 j} \equiv \sigma_{0}$ ) and a symmetric beam with equal emittances in both planes $\left(\varepsilon_{j} \equiv \varepsilon\right)$. Under these assumptions, the depressed phase advances $\sigma_{j}$ are also equal in both planes $\left(\sigma_{j} \equiv \sigma\right)$ and parametrization cases 2 and 3 of Table I are identical. First, parametrization cases 1 (specified $\kappa_{j}, Q$ and $\sigma$ ) and 2 (specified $\kappa_{j}, \varepsilon$, and $\sigma$ ) are examined in Sec. VA. Both of these cases have specified depressed phase advance $\sigma$ and represent the most "natural" parametrization of the IM method. Then results in Sec. VA are extended in Sec. V B to illustrate how the IM method can be applied to parametrization case 0 (specified $\kappa_{j}, Q$, and $\varepsilon$ ) with unspecified $\sigma$ while circumventing practical implementation difficulties.

For simplicity, we further restrict our examples to periodic solenoidal and quadrupole doublet focusing lattices with piecewise constant focusing functions $\boldsymbol{\kappa}_{j}(s)$ as illustrated in Fig. 1. Solenoidal focusing has $\kappa_{x}(s)=\kappa_{y}(s)$, and alternating gradient quadrupole focusing has $\kappa_{x}(s)=$ $-\kappa_{y}(s)$. For both the solenoid and quadrupole lattices illustrated, $\eta \in(0,1]$ is the fractional occupancy of the focusing elements in the lattice period $L_{p}$. The focusing strength of the elements is taken to be $\left|\kappa_{j}\right|=\hat{\kappa}=$ const within the axial extent of the optics and zero outside. For solenoids, $\kappa_{j}=\hat{\kappa}>0$ in the focusing element; and for quadrupoles, $\kappa_{x}=-\kappa_{y}=\hat{\kappa}>0$ in the focusing-in- $x$ element of the doublet, and $\kappa_{x}=-\kappa_{y}=-\hat{\kappa}<0$ in the defocusing-in- $x$ element. The free drift between solenoids has axial length $d=(1-\eta) L_{p}$. For quadrupole doublet
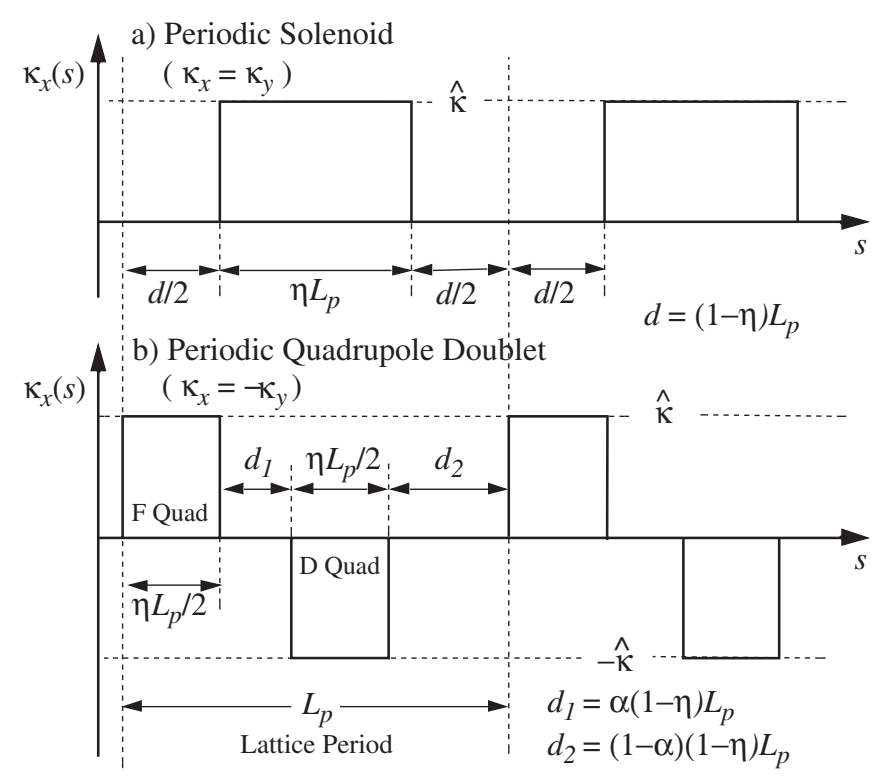

FIG. 1. Periodic (a) solenoid and (b) quadrupole focusing lattices with piecewise constant $\kappa_{j}$.

focusing, the two drift distances $d_{1}=\alpha(1-\eta) L_{p}$ and $d_{2}=(1-\alpha)(1-\eta) L_{p}$ separating focusing and defocusing quadrupoles can be unequal (i.e., $d_{1} \neq d_{2}$ ). A syncopation parameter $\alpha \in[0,1]$ provides a measure of this asymmetry. Without loss of generality, the lattice can always be relabeled to take $\alpha \in[0,1 / 2]$, with $\alpha=0$ corresponding to the focusing and defocusing lenses touching each other $\left[d_{1}=0\right.$ and $\left.d_{2}=(1-\eta) L_{p}\right]$. The special case of $\alpha=1 / 2$ corresponding to equally spaced drifts $\left[d_{1}=\right.$ $\left.d_{2}=(1-\eta) L_{p} / 2\right]$ is defined as a "FODO" lattice. These focusing lattices are discussed in more detail in Ref. [3], including a description of how the focusing strength parameter $\hat{\kappa}$ is related to magnetic and/or electric fields of physical realizations of the focusing elements.

As discussed in Sec. II, for general lattices the scale of the focusing functions $\kappa_{j}$ can be set by the undepressed phase advances $\sigma_{0 j}$ using Eq. (10). For the piecewise constant $\kappa_{j}$ defined in Fig. 1, explicit calculations [3] show that the focusing strength $|\hat{\kappa}|$ is related to $\sigma_{0}$ by the constraint equations:

$$
\cos \sigma_{0}= \begin{cases}\cos (2 \Theta)-\frac{1-\eta}{\eta} \Theta \sin (2 \Theta), & \text { solenoidal focusing } \\ \cos \Theta \cosh \Theta & \text { quadrupole focusing. } \\ +\frac{1-\eta}{\eta} \Theta(\cos \Theta \sinh \Theta-\sin \Theta \cosh \Theta) & \\ -2 \alpha(1-\alpha) \frac{(1-\eta)^{2}}{\eta^{2}} \Theta^{2} \sin \Theta \sinh \Theta & \end{cases}
$$

Here, for both solenoidal and quadrupole focusing lattices, $\Theta=\sqrt{|\hat{\kappa}|} \eta L_{p} / 2$. In the analysis that follows, Eq. (39) is employed to numerically calculate $\Theta$ for a specified value of $\sigma_{0}$, and then $\hat{\kappa}$ is calculated in terms of other specified lattice parameters as $|\hat{\kappa}|=4 \Theta^{2} /\left(\eta L_{p}\right)^{2}$. The undepressed phase advance $\sigma_{0}$ is measured in degrees per lattice period. Integrations of needed principal orbits to implement the IM method are carried out with the initial conditions $\left(s=s_{i}\right)$ corresponding to the axial midpoint of drifts separating focusing elements. 



FIG. 2. (Color) Example matched envelope solutions for (a) solenoidal, (b) FODO ( $\alpha=1 / 2)$ quadrupole, and (c) syncopated ( $\alpha=0.1$ ) quadrupole focusing lattice. Parameters for all cases are: $L_{p}=0.5 \mathrm{~m}, \eta=0.5, \sigma_{0}=80^{\circ}, Q=4 \times 10^{-4}$, and $\varepsilon=$ $50 \mathrm{~mm}$ mrad. These parameters yield $\sigma / \sigma_{0}=0.3144,0.3093$, and 0.3099 for (a), (b), and (c).

Typical matched envelope solutions $r_{j}$ are shown for one lattice period in Fig. 2 for solenoid, FODO $(\alpha=1 / 2)$ quadrupole, and syncopated $(\alpha \neq 1 / 2)$ quadrupole focusing lattices. Scaled $x$-plane lattice focusing functions $\kappa_{x}$ are shown superimposed. Excursions of the matched envelope functions are in-phase for solenoidal focusing $\left(r_{x}=\right.$ $\left.r_{y}\right)$ because the applied focusing is plane symmetric $\left(\kappa_{x}=\right.$ $\kappa_{y}$ ). In contrast, for quadrupole focusing, the antisymmetric plane focusing $\left(\kappa_{x}=-\kappa_{y}\right)$ results in out of phase envelope flutter in each plane (focus-defocus) leading to net focusing over the full lattice period in both planes.
Expected symmetries of the matched solutions are present for both the solenoidal and quadrupole focusing lattices (see Appendix A). For the quadrupole solutions, note that the FODO case exhibits a higher degree of subperiod symmetry than the syncopated case. Leading-order terms of an analytical perturbation theory for the matched beam envelope solution [7] can be applied in the limit $\sigma \rightarrow 0$ to show that the envelope excursions (flutter) scale as

$$
\begin{aligned}
& \frac{\operatorname{Max}\left[r_{x}\right]}{\overline{r_{x}}}-1 \\
& \simeq \begin{cases}\frac{\left(1-\cos \sigma_{0}\right)(1-\eta)(1-\eta / 2)}{6}, & \text { solenoidal focusing } \\
\frac{\left(1-\cos \sigma_{0}\right)^{1 / 2}(1-\eta / 2)}{2^{3 / 2}(1-2 \eta / 3)^{1 / 2}}, & \text { FODO quadrupole focusing. }\end{cases}
\end{aligned}
$$

Equation (40) shows that for solenoidal focusing the matched envelope flutter increases with decreasing lattice occupancy $\eta$ and increasing focusing strength $\sigma_{0}$. In contrast, for FODO quadrupole focusing the flutter depends weakly on $\eta$ (the variation of $\operatorname{Max}\left[r_{x}\right] / \overline{r_{x}}-1$ in $\eta$ has a maximum range of 0.07) and more strongly on $\sigma_{0}$ (variation of 0.5 ). Envelope flutter changes only weakly when space-charge strength is reduced (i.e., $\sigma / \sigma_{0}$ increased).

Although the system symmetries assumed simplify interpretation of the matched envelope solutions obtained in the examples, we note that the numerical methods employed in calculation of the principal orbits functions and any necessary constraint equations are not structured to take advantage of the symmetries of the matched solutions. Because of this, the examples provide a better guide on the performance of the IM method in situations where there are lesser degrees of system symmetry. MATHEMATICA [16] based programs used in the examples have been archived $[17,18]$. These programs can be easily adapted to more complicated lattices.

\section{A. Case 1 and 2 parametrizations}

The IM method described in Sec. IV is applied with $\sigma^{i}=\sigma$ specified and the unknown parameters of the $i$ th iteration $\varepsilon^{i}$ (case 1: $Q$ and $\sigma$ specified) or $Q^{i}$ (case 2: $\varepsilon$ and $\sigma$ specified) calculated from the constraint equations (34) and (35). The continuous focusing approximation envelope radii $\overline{r_{j}}$ used in the seed $(i=0)$ iterations are calculated from Eq. (30) (case 1) and Eq. (28) (case 2). The number of iterations needed to achieve a $10^{-6}$ fractional envelope tolerance [see Eq. (38)] are presented in Fig. 3 as a function of $\sigma_{0}$ and $\sigma / \sigma_{0}$ for solenoidal, FODO quadrupole, and syncopated quadrupole focusing lattices employing both case 1 and case 2 parametrization methods. In Fig. 4, iterations corresponding to one data point in Fig. 3 (case 2 solenoidal focusing) are shown. This example shows a result typical for lower to intermediate values of $\sigma_{0}$, where the seed iteration $r_{j}^{0}$ is fairly close to the matched solution and the first iteration correction $r_{j}^{1}$ closely tracks the 


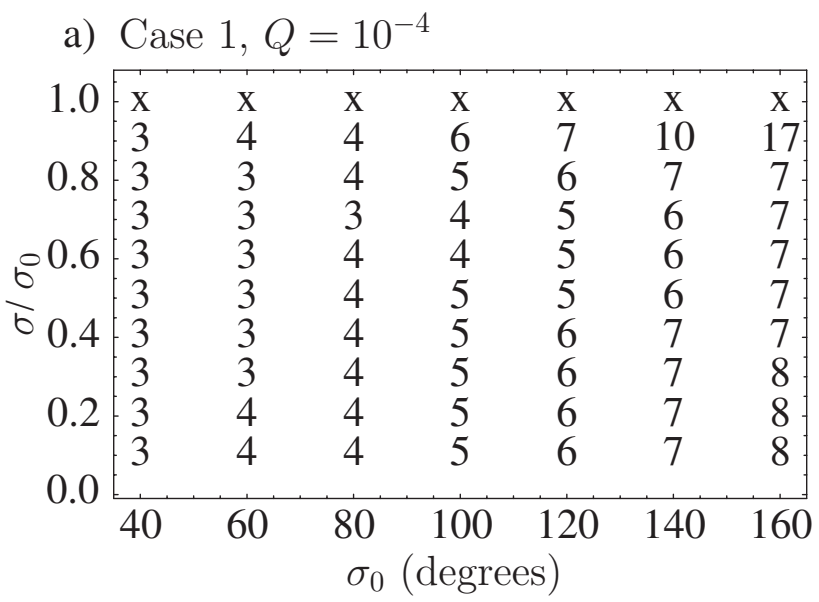

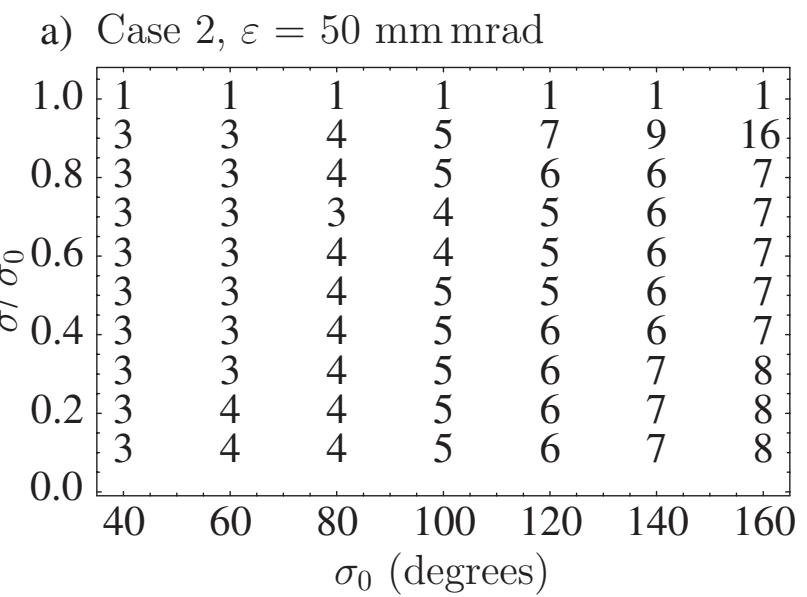

b) Case $1, Q=10^{-4}$

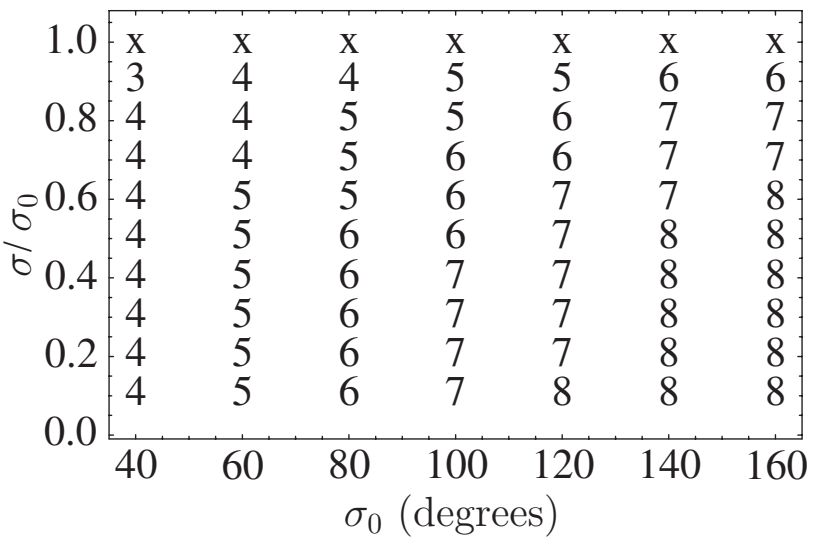

b) Case 2, $\varepsilon=50 \mathrm{~mm}$ mrad

\begin{tabular}{|c|c|c|c|c|c|c|c|}
\hline \multirow[t]{2}{*}{1.0} & 1 & 1 & 1 & 1 & 1 & 1 & 1 \\
\hline & 3 & 4 & 4 & 5 & 5 & 6 & 6 \\
\hline \multirow{2}{*}{0.8} & 4 & 4 & 5 & 5 & 6 & 7 & 7 \\
\hline & 4 & 4 & 5 & 6 & 7 & 7 & 7 \\
\hline \multirow{2}{*}{$0^{0.6}$} & 4 & 5 & 5 & 6 & 7 & 7 & o \\
\hline & 4 & 5 & 6 & 6 & 7 & 8 & 8 \\
\hline \multirow[t]{2}{*}{${ }^{6} 0.4$} & 4 & 5 & 6 & 7 & 7 & 8 & o \\
\hline & 4 & 5 & 6 & 7 & 7 & 8 & 8 \\
\hline \multirow[t]{2}{*}{0.2} & 4 & 5 & 6 & 7 & 7 & 8 & 8 \\
\hline & 4 & 5 & 6 & 7 & 8 & 8 & 0 \\
\hline & 40 & & 8 & 0 & 120 & 140 & 16 \\
\hline
\end{tabular}

c) Case $1, Q=10^{-4}$

\begin{tabular}{|c|c|c|c|c|c|c|c|}
\hline 1.0 & $x$ & $\mathrm{x}$ & $\mathrm{x}$ & $\mathrm{x}$ & $\mathrm{X}$ & $\mathrm{x}$ & $\mathrm{x}$ \\
\hline & 4 & 4 & 5 & 5 & 6 & 7 & 8 \\
\hline 0.8 & 3 & 4 & 4 & 5 & 6 & 6 & 7 \\
\hline & 4 & 4 & 5 & 5 & 6 & 6 & 7 \\
\hline 0.6 & 4 & 4 & 5 & 6 & 6 & 7 & 7 \\
\hline & 4 & 4 & 5 & 6 & 6 & 7 & 7 \\
\hline${ }^{\circ} 0.4$ & 4 & 5 & 5 & 6 & 7 & 7 & 7 \\
\hline & 4 & 5 & 5 & 6 & 7 & 7 & 8 \\
\hline 0.2 & 4 & 5 & 6 & 6 & 7 & 7 & 8 \\
\hline & 4 & 5 & 6 & 6 & 7 & 7 & 8 \\
\hline & 40 & 60 & 80 & 100 & 120 & 140 & 16 \\
\hline
\end{tabular}

c) Case 2, $\varepsilon=50 \mathrm{~mm} \operatorname{mrad}$

\begin{tabular}{|c|c|c|c|c|c|c|c|}
\hline \multirow{2}{*}{1.0} & 1 & 1 & 1 & 1 & 1 & 1 & 1 \\
\hline & 3 & 4 & 5 & 5 & 6 & 7 & 7 \\
\hline \multirow{2}{*}{0.8} & 3 & 4 & 4 & 5 & 6 & 6 & 7 \\
\hline & 3 & 4 & 5 & 5 & 6 & 6 & 7 \\
\hline \multirow{2}{*}{$8^{0.6}$} & 4 & 4 & 5 & 5 & 6 & 7 & 7 \\
\hline & 4 & 4 & 5 & 6 & 6 & 7 & 7 \\
\hline \multirow{2}{*}{${ }^{\circ} 0.4$} & 4 & 5 & 5 & 6 & 6 & 7 & 8 \\
\hline & 4 & 5 & 5 & 6 & 7 & 7 & 8 \\
\hline \multirow[t]{2}{*}{0.2} & 4 & 5 & 5 & 6 & 7 & 7 & 8 \\
\hline & 4 & 5 & 5 & 6 & 7 & 7 & 8 \\
\hline & 40 & 60 & 80 & 100 & 120 & 140 & 160 \\
\hline
\end{tabular}

FIG. 3. Number of IM iterations needed to achieve a tol $=10^{-6}$ fractional error tolerance matched envelope solution for (a) solenoidal, (b) FODO ( $\alpha=1 / 2)$ quadrupole, and (c) syncopated ( $\alpha=0.1$ ) quadrupole focusing lattices as a function of $\sigma_{0}$ (for $\left.\sigma_{0}=40^{\circ}, 60^{\circ}, 80^{\circ}, \ldots, 160^{\circ}\right)$ and $\sigma / \sigma_{0}$ (for $\sigma / \sigma_{0}=0.1,0.2,0.3, \ldots, 1.0$ ). The left column corresponds to the parametrization case 1 method with $Q=10^{-4}$ (unachievable limit points marked $\mathrm{x}$ ) and the right column corresponds to the parametrization case 2 method with $\varepsilon=50 \mathrm{~mm}$ mrad. Other lattice parameters are $L_{p}=0.5 \mathrm{~m}$ and $\eta=0.5$ for (a), (b), and (c).

matched solution to within a percent local fractional error. Higher values of $\sigma_{0}$ and more complicated lattices result in both seed iterations farther from the matched solution and less rapid convergence with iteration number.

The data in Fig. 3 show that the IM method converges rapidly to small tolerances over a broad range of applied focusing $\left(\sigma_{0}\right)$ and space-charge $\left(\sigma / \sigma_{0}\right)$ strength. Not surprisingly, stronger focusing strength (i.e., increasing $\sigma_{0}$ ) requires more iterations for both solenoidal and quadrupole focusing at the fixed value of lattice occupancy $\eta$ employed. Also, lesser degrees of lattice symmetry result in more iterations being necessary for convergence (e.g., 


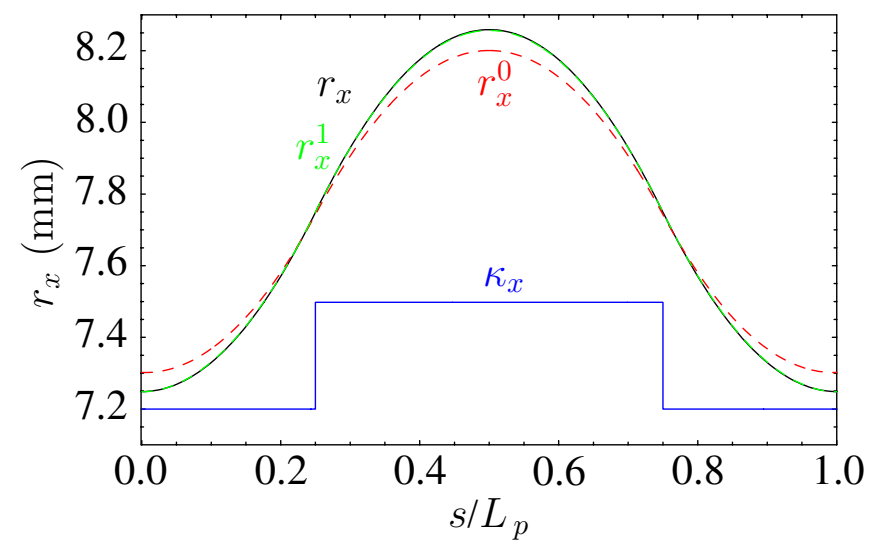

FIG. 4. (Color) For solenoidal focusing: converged matched envelope solution (black) with $r_{x}=r_{y}$, the first seed iteration $r_{x}^{0}$ (red), and the second iteration $r_{x}^{1}$ (green). System parameters are: $L_{p}=0.5 \mathrm{~m}, \eta=0.5, \sigma_{0}=80^{\circ}, \varepsilon=50 \mathrm{~mm} \mathrm{mrad}$, and $\sigma / \sigma_{0}=0.3$.
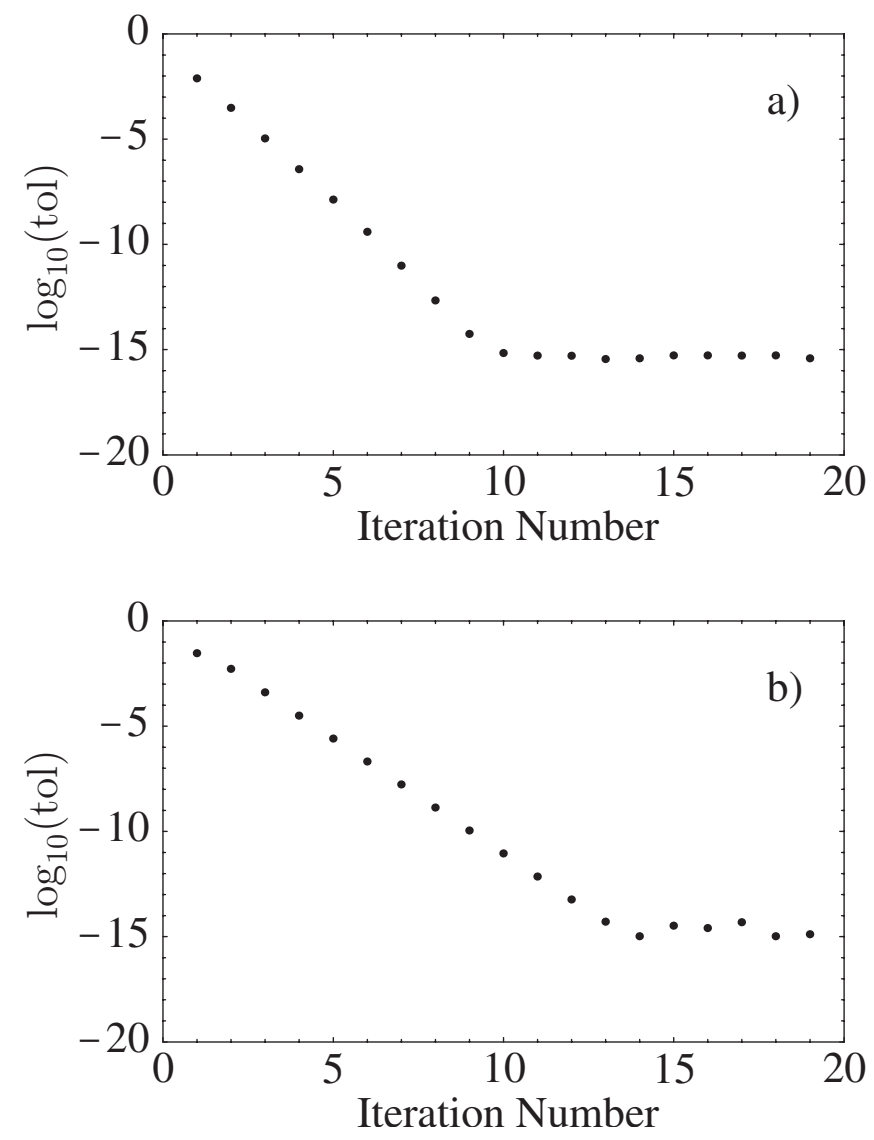

FIG. 5. Log of fractional error tolerance (tol) achieved for matched envelope solutions versus number of IM iterations for (a) solenoidal and (b) FODO ( $\alpha=1 / 2)$ quadrupole focusing lattices. Solutions are generated using case 2 methods for system parameters: $L_{p}=0.5 \mathrm{~m}, \eta=0.5, \sigma_{0}=80^{\circ}, \varepsilon=50 \mathrm{~mm} \mathrm{mrad}$, and $\sigma / \sigma_{0}=0.2$ [corresponding to $Q \simeq 6.700 \times 10^{-4}$ and $Q \simeq$ $6.561 \times 10^{-4}$ for (a) and (b)]. lattice convergence rate order: solenoidal, FODO quadrupole, syncopated quadrupole). Iterations required appear to depend only weakly on space-charge strength $\left(\sigma / \sigma_{0}\right)-$ except for solenoidal focusing lattices with very high $\sigma_{0}$ where required iterations become abruptly larger for weak space charge with $\sigma / \sigma_{0}$ close to unity. Even parameters deep within the regime of strong envelope instability [3] converge rapidly. Points for $\sigma / \sigma_{0}=1$ are eliminated in the case 1 examples because the perveance $Q$ is held to a fixed, finite value and this limit would correspond to a matched beam envelope with infinite cross-sectional area. Conversely, for the limit $\sigma / \sigma_{0}=1$ in the case 2 examples, only one iteration is required for convergence to a finite solution because for zero space-charge strength the trial seed iteration generated by Eq. (32) for $i=0$ corresponds to the exact matched envelope to numerical error (i.e., when $Q^{0}=0$ the $C_{j}^{0}$ and $S_{j}^{0}$ are the principal undepressed particle orbits which generate the matched envelope of the undepressed beam). The IM method applies for extremely strong space charge with $\sigma / \sigma_{0} \ll 0.1$, but probing the limit $\sigma \rightarrow 0$ requires careful analysis of various terms in the formulation presented in Sec. IV.

Complementary to Fig. 3, the decrease in the log of the fractional tolerance [see Eq. (38)] achieved with iteration number is plotted in Fig. 5 for solenoidal and FODO quadrupole focusing lattices for one set of system parameters. The matched envelopes are calculated using the case 2 methods. Case 1 methods and other system parameters yield similar results to those presented. We find that the IM method converges rapidly, with the fractional tolerance achieved increasing by 1 to 2 orders of magnitude per iteration till saturating at a value reflecting the precision of numerical calculations carried out $\left(\sim 10^{-15}\right.$ fractional accuracy for the examples in Fig. 5).

\section{B. Case 0 parametrization}

In parametrization case 0 , the matched envelope functions $r_{j}$ are specified by $\kappa_{j}, Q$, and $\varepsilon_{j}$. For the $i$ th iteration, the depressed phase advances $\sigma_{j}^{i}$ needed to calculate the iteration envelope functions $r_{j}^{i}$ are most simply calculated using Eq. (33). Unfortunately, this simple method can fail if space charge is strong and iterations produce envelope corrections where the radial cross section of the beam is compressed sufficiently relative to the actual matched envelope solution over the lattice period. Compressive overcorrections can produce iteration principal orbits that are depressed below zero phase advance (i.e., $\sigma_{j}^{i}=0$ ). In this situation, $\sigma_{j}^{i}$ becomes complex and we find that Eq. (32) can fail to generate an iteration closer to the desired matched envelope solution.

To better understand where the problem described above can occur, a simple continuous focusing estimate (see Sec. III B) is applied. Taking $\kappa_{j}=\left(\sigma_{0} / L_{p}\right)^{2}$ and $\varepsilon_{j}=\varepsilon$, we estimate the envelope compression factor $f$ needed to 
fully depress particle orbits within the matched envelope. A particle moving within the continuous matched envelope $r_{j}=\overline{r_{b}}$ has depressed phase advance $\left(\sigma / L_{p}\right)^{2}=$ $\left(\sigma_{0} / L_{p}\right)^{2}-Q /{\overline{r_{b}}}^{2}$. Replacing $\overline{r_{b}} \rightarrow f \overline{r_{b}}$ and $\sigma \rightarrow 0$ in this phase advance formula gives

$$
f=\frac{\sqrt{2}}{\sqrt{1+\sqrt{1+4\left[\sigma_{0} \varepsilon /\left(Q L_{p}\right)\right]^{2}}}}
$$

But for continuous focusing, we have [3]

$$
\frac{\sigma_{0} \varepsilon}{Q L_{p}}=\frac{\left(\sigma / \sigma_{0}\right)}{1-\left(\sigma / \sigma_{0}\right)^{2}} .
$$

Together, Eqs. (41) and (42) show that $f=0.99,0.95$, and 0.90 (corresponding to $\sim 1 \%, 5 \%$, and $10 \%$ compressive overcorrections) will produce fully depressed particle orbits for $\sigma / \sigma_{0}<0.14,0.31$, and 0.44 . Numerically analyzed examples below indicate that this problem can occur in periodic focusing lattices for more moderate space charge and compression factors than these continuous focusing estimates suggest.

The parameter region where the IM method can be applied using the "conventional" case 0 procedure for example periodic solenoid and FODO quadrupole lattices is illustrated in Fig. 6. The region of applicability corresponds to parameters where Eq. (33) can be employed to calculate the iteration depressed phases advances $\sigma_{j}^{i}$ without obtaining complex values. Iterations necessary to achieve tolerance are plotted as a function of $\sigma_{0}$ and $\sigma / \sigma_{0}$. Rather than plotting results in terms of the perveance $Q$, Eq. (14) was used to calculate $\sigma / \sigma_{0}$ from the matched envelope functions and system parameters to better quantify the relative space-charge strength where the method fails. Values of $Q$ were chosen to uniformly distribute points in $\sigma / \sigma_{0}$. Note that the IM method works with the simple initial seed iteration when space charge is moderate to weak $\left(0.6<\sigma / \sigma_{0} \leq 1\right)$ but abruptly fails with increasing space charge $\left(\sigma / \sigma_{0}<0.6\right)$. Near the point of failure, convergence becomes slow (iteration counts for the examples in Fig. 6 can become thousands if points are chosen sufficiently close to the failure region).

Several alternative methods were attempted to render the IM method applicable to all case 0 parameters with arbitrary space-charge strength. We formulate these methods without reference to a specific lattice or taking $\sigma_{0 x}=\sigma_{0 y}$ and $\varepsilon_{x}=\varepsilon_{y}$ to better reflect general case 0 applications. First, rather than employing Eq. (33) to calculate the depressed phase advance $\sigma_{j}^{i}$ of the iteration, the integral formula (14) is applied with the envelope functions of the previous $i-1$ iteration with

$$
\sigma_{j}^{i}=\varepsilon_{j} \int_{s_{i}}^{s_{i}+L_{p}} \frac{d s}{\left[r_{j}^{i-1}(s)\right]^{2}} .
$$

\begin{tabular}{|c|c|c|c|c|c|c|c|}
\hline \multirow{2}{*}{1.0} & 1 & 1 & 1 & 1 & 1 & 1 & 1 \\
\hline & 3 & 4 & 4 & 5 & 5 & 6 & 9 \\
\hline \multirow[t]{2}{*}{0.8} & 5 & 6 & 7 & 8 & 9 & 9 & 10 \\
\hline & 10 & 12 & 14 & 15 & 16 & 16 & 15 \\
\hline$\bullet^{\circ} 0.6$ & 53 & 67 & 77 & 82 & 81 & 68 & 44 \\
\hline \multirow{3}{*}{ b 0.4} & $\mathrm{X}$ & $\mathrm{X}$ & $\mathrm{X}$ & $\mathrm{X}$ & $\mathrm{X}$ & $\mathrm{X}$ & $\mathrm{X}$ \\
\hline & X & $\mathrm{X}$ & X & X & $\mathrm{X}$ & X & $\mathrm{X}$ \\
\hline & $\mathrm{X}$ & $\mathrm{X}$ & $\mathrm{X}$ & $\mathrm{X}$ & $\mathrm{X}$ & $\mathrm{X}$ & $\mathrm{X}$ \\
\hline \multirow[t]{2}{*}{0.2} & $\mathrm{X}$ & $\mathrm{X}$ & $\mathrm{X}$ & $\mathrm{X}$ & $\mathrm{X}$ & $\mathrm{X}$ & $\mathrm{X}$ \\
\hline & $\mathrm{X}$ & $\mathrm{X}$ & $\mathrm{X}$ & $\mathrm{X}$ & $\mathrm{X}$ & $\mathrm{X}$ & $\mathrm{X}$ \\
\hline 0.0 & 40 & 60 & 80 & 100 & 120 & 140 & 160 \\
\hline
\end{tabular}

The anticipation is that $\sigma_{j}^{i}$ calculated from Eq. (43) should a)

\begin{tabular}{|c|c|c|c|c|c|c|c|}
\hline \multirow[t]{2}{*}{1.0} & 1 & 1 & 1 & 1 & 1 & 1 & 1 \\
\hline & 5 & 6 & 6 & 6 & 5 & 6 & 10 \\
\hline \multirow{2}{*}{0.8} & 9 & 9 & 9 & 9 & 8 & 7 & 8 \\
\hline & 17 & 18 & 18 & 17 & 14 & 11 & 9 \\
\hline \multirow{2}{*}{$b^{0} 0.6$} & 90 & 89 & 76 & 57 & 38 & 22 & 15 \\
\hline & $\mathrm{X}$ & $\mathrm{X}$ & $\mathrm{X}$ & X & $\mathrm{X}$ & X & 63 \\
\hline \multirow[t]{2}{*}{$\begin{array}{ll}6 & 0.4\end{array}$} & $\mathrm{X}$ & $\mathrm{X}$ & $\mathrm{X}$ & $X$ & $\mathrm{X}$ & $\mathrm{X}$ & $\mathrm{X}$ \\
\hline & X & $\mathrm{X}$ & $\mathrm{X}$ & $\mathrm{X}$ & $X$ & $X$ & $\mathrm{X}$ \\
\hline \multirow{2}{*}{0.2} & $\mathrm{X}$ & $\mathrm{X}$ & $\mathrm{X}$ & $\mathrm{X}$ & $\mathrm{X}$ & $\mathrm{X}$ & $\mathrm{X}$ \\
\hline & $\mathrm{X}$ & $\mathrm{X}$ & $\mathrm{X}$ & $\mathrm{X}$ & $\mathrm{X}$ & $\mathrm{X}$ & $\mathrm{X}$ \\
\hline 0.0 & 40 & 60 & 80 & 100 & $\begin{array}{l}120 \\
\mathrm{es})\end{array}$ & 140 & 160 \\
\hline
\end{tabular}

b)

FIG. 6. Number of conventional IM iterations needed in case 0 to achieve a tol $=10^{-6}$ fractional error tolerance matched envelope solution for (a) solenoidal and (b) FODO $(\alpha=1 / 2)$ quadrupole lattices as a function of $\sigma_{0}$ (for $\sigma_{0}=40^{\circ}, 60^{\circ}, 80^{\circ}$, $\ldots, 160^{\circ}$ ) and $\sigma / \sigma_{0}$ (for $\sigma / \sigma_{0}=0.1,0.2,0.3, \ldots, 1.0$ ). System parameters are: $L_{p}=0.5 \mathrm{~m}, \eta=0.5$, and $\varepsilon=50 \mathrm{~mm} \mathrm{mrad}$. Parameters where the method fails are marked $\mathrm{x}$.

be sufficiently close to the actual depressed phase advance $\sigma_{j}$ of the converged solution to correct the problem. Unfortunately, this method, when applied to the example solenoid and quadrupole lattices, results in systematic convergence to unphysical solutions. Replacing Eq. (43) with an "under-relaxed" average over previous iterations might address this problem. In cases where complex phase advances resulted, various other simple replacements of Eq. (33) have been attempted without obtaining satisfactory results.

Several alternative procedures extend applicability to general case 0 parameters. First, slowly increasing the perveance $Q$ from some sufficiently small (or zero) value while implementing the conventional case 0 iteration method using Eq. (33) proves workable in our tests. In this scheme, if Eq. (33) fails (i.e., produces unphysical complex values for $\sigma_{j}^{i}$ ), then $Q$ is adaptively decreased while iterating until the formula becomes valid before 
increasing $Q$ again toward the target value. For strong space charge this procedure can result in many iterations being necessary for convergence because small increases in $Q$ were required in various test cases examined. It is also difficult to determine optimal increments to increase the perveance-which complicates practical code development and can limit the range of method applicability.

Another, simpler to implement, alternative procedure is formulated by combining the Sec. VA method for solving case 1 parametrizations with numerical root finding. In this "hybrid" procedure, the emittances $\varepsilon_{j}$ calculated from the $x$ - and $y$-plane constraint equations (23) are regarded as an undetermined function of the $\sigma_{j}$ [i.e., $\left.\varepsilon_{j}\right|_{\text {specified }}=$ $\left.\varepsilon_{j}\left(\sigma_{x}, \sigma_{y}\right)\right]$ and trial matched envelope solutions $r_{j}$ are rapidly calculated to tolerance using matched envelopes obtained with case 1 methods for specified (guessed) values of the $\sigma_{j}$. Numerical root finding can be employed to refine the guessed values for the $\sigma_{j}$ to obtain the values of $\sigma_{j}$ consistent with the target values of $\varepsilon_{j}$. Because the $\varepsilon_{j}\left(\sigma_{x}, \sigma_{y}\right)$ are smooth, monotonic functions of the $\sigma_{j}$ for $0<\sigma_{j}<\sigma_{0 j}$, the consistent values of the $\sigma_{j}$ can be found with relatively small numbers of root finding iterations. This is particularly true for plane-symmetric systems ( $\sigma_{0 j}=\sigma_{0}$ and $\varepsilon_{j}=\varepsilon$ ) because one-dimensional root finding can be employed.

The total number of two-dimensional (i.e., the calculations do not assume plane symmetry) iterations needed to implement this hybrid method for case 0 is shown in Fig. 7 for example periodic solenoid and FODO quadrupole lattices. Here, the total iteration number represents the sum of all iterations needed to calculate the emittances to a specified fractional tolerance while calculating all trial matched envelope solutions to a separate specified tolerance over all two-dimensional root finding steps. The same lattices and presentation methods used in Fig. 6 are employed to aid comparisons. Note that the full case 0 parameter space is accessible in this procedure with only relatively modest total iteration counts in spite of the additional numerical work resulting from the root finding. A secantlike multidimensional root finding method is employed [16]. Note that only two-dimensional root finding is necessary in contrast to four-dimensional root finding associated with conventional procedures for constructing matched envelope solutions by finding appropriate initial envelope coordinates and angles. Initial root finding iterations are seeded using continuous focusing model estimates for $\sigma_{j}$ calculated from Eq. (28) using the seed values of $\overline{r_{j}}$. Subsequent root finding steps in $\sigma_{j}$ employ the previous step matched envelopes as a seed envelope in the case 1 iterations. For small root finding steps in $\sigma_{j}$ this previous step seeding saves considerable numerical work. Only one iteration is necessary for the limit points with $\sigma / \sigma_{0}=1$ because for zero space-charge strength the trial seed iteration is exact to numerical error. Iteration counts at fixed $\sigma_{0}$

\begin{tabular}{|c|c|c|c|c|c|c|c|}
\hline \multirow{2}{*}{1.0} & 1 & 1 & 1 & 1 & 1 & 1 & 1 \\
\hline & 9 & 21 & 14 & 14 & 24 & 30 & 56 \\
\hline \multirow[t]{2}{*}{0.8} & 9 & 9 & 10 & 15 & 16 & 37 & 23 \\
\hline & 9 & 9 & 9 & 12 & 32 & 18 & 21 \\
\hline \multirow{2}{*}{ ○0.6 } & 9 & 9 & 10 & 14 & 15 & 16 & 19 \\
\hline & 9 & 9 & 10 & 15 & 15 & 16 & 21 \\
\hline \multirow{2}{*}{$\begin{array}{ll}b & 0.4\end{array}$} & 9 & 9 & 10 & 11 & 16 & 20 & 19 \\
\hline & 9 & 9 & 10 & 11 & 16 & 19 & 32 \\
\hline \multirow[t]{2}{*}{0.2} & 7 & 10 & 10 & 11 & 16 & 17 & 20 \\
\hline & 7 & 10 & 10 & 11 & 12 & 13 & 18 \\
\hline 0.0 & 40 & 60 & $\begin{array}{r}80 \\
\sigma\end{array}$ & $\begin{array}{l}100 \\
\text { deg }\end{array}$ & & 140 & 160 \\
\hline
\end{tabular}

b)

\begin{tabular}{|c|c|c|c|c|c|c|c|}
\hline \multirow{2}{*}{1.0} & 1 & 1 & 1 & 1 & 1 & 1 & 1 \\
\hline & 9 & 16 & 16 & 19 & 18 & 22 & 22 \\
\hline \multirow[t]{2}{*}{0.8} & 10 & 23 & 17 & 18 & 19 & 20 & 23 \\
\hline & 10 & 15 & 15 & 18 & 20 & 21 & 23 \\
\hline \multirow{2}{*}{$b^{0} 0.6$} & 10 & 15 & 15 & 18 & 21 & 23 & 23 \\
\hline & 10 & 17 & 16 & 16 & 19 & 38 & 24 \\
\hline \multirow{2}{*}{${ }^{b} 0.4$} & 10 & 15 & 16 & 16 & 19 & 22 & 22 \\
\hline & 10 & 13 & 14 & 17 & 19 & 20 & 22 \\
\hline \multirow[t]{2}{*}{0.2} & 10 & 11 & 16 & 17 & 17 & 20 & 22 \\
\hline & 10 & 11 & 12 & 17 & 16 & 18 & 18 \\
\hline & 40 & & 80 & & 120 & 140 & 160 \\
\hline
\end{tabular}

FIG. 7. Number of total hybrid IM iterations needed for case 0 using tol $=10^{-6}$ fractional error tolerance case 1 matched envelope solutions and root found $\varepsilon_{j}$ with $10^{-4}$ fractional accuracy from specified values for (a) solenoidal and (b) FODO $(\alpha=1 / 2)$ quadrupole lattices. The same system parameters and presentation format are employed as in Fig. 6.

likely increase and decrease in $\sigma / \sigma_{0}$ due to approximate iteration seed guesses being (accidentally) farther and nearer to the actual root than in other cases. If the plane symmetries are employed (i.e., using $\varepsilon_{x}=\varepsilon_{y}$ and $\sigma_{x}=$ $\sigma_{y}$ ), then total iterations required can be further reduced. Matched envelopes for general case 0 parameters can also be calculated in similar values of total iterations by analogously combining case 2 methods with numerical root finding. In this case 2 hybrid method, values of $\sigma_{j}$ consistent with specified values of $Q$ are calculated using the two components of Eq. (23) [i.e., set $Q \rightarrow Q_{j}$ in the $j=x$ and $y$ components of Eq. (23) and then root solve for $\sigma_{j}$ consistent with $\left.Q=Q_{j}\left(\sigma_{x}, \sigma_{y}\right)\right]$.

\section{CONCLUSIONS}

An iterative matching (IM) method for numerical calculation of the matched beam envelope solutions to the KV 
equations has been developed. The method is based on orbit consistency conditions between depressed particle orbits within a KV beam distribution and the envelope of orbits making up the distribution. Application of the IM method in simplest form requires numerical solution of linear ordinary differential equations describing principal particle orbits over one lattice period and the calculation of a few axillary integrals over the lattice period. A large basin of convergence enables seeding of the iterations with a simple trial solution that takes into account both the envelope flutter driven by the applied focusing lattice and leading-order space-charge defocusing forces. All cases of envelope parametrizations can be employed, but the method is most naturally expressed, and highly convergent, when employing the depressed particle phase advances $\sigma_{j}$ as parameters - which also corresponds to a natural choice of parameters to employ for enhanced physics understanding. Virtues of the IM method are: it is straightforward to code and applicable to periodic focusing lattices of arbitrary complexity; it is efficient for arbitrary space-charge intensity; and it works for all physically achievable system parameters - even in bands of parametric envelope instability where conventional matching procedures can fail.

\section{ACKNOWLEDGMENTS}

The authors wish to thank J. J. Barnard and A. Friedman for useful discussions. This research was performed under the auspices of the U.S. Department of Energy at the Lawrence Livermore and Lawrence Berkeley National Laboratories under Contracts No. W-7405-Eng-48 and No. DE-AC03-76SF0098.

\section{APPENDIX: MATCHED ENVELOPE SYMMETRIES FOR QUADRUPOLE DOUBLET AND SOLENOIDAL FOCUSING}

Consider a periodic quadrupole doublet lattice [3] focusing a beam with equal $x$ - and $y$-plane emittances (i.e., $\varepsilon_{x}=$ $\varepsilon_{y}$ ). To concretely define doublet focusing, we assume that an $s$-coordinate origin can be chosen such that the lattice focusing functions $\kappa_{j}(s)$ satisfy

$$
\kappa_{j}(s)=-\kappa_{j}(-s)
$$

in addition to the general quadrupole lattice symmetry $\kappa_{x}=-\kappa_{y}$. This doublet focusing symmetry is consistent with focusing/defocusing elements with axial structure (i.e., including fringe fields) if both the focusing and defocusing elements are realized by identical hardware assemblies with equal field excitations appropriately arranged in a regular lattice via symmetry operations (i.e., translations and rotations). Note that $s=0$ corresponds to the axial location of the drift between two successive quadrupoles in the periodic lattice (for cases where a finite fringe field extends into the drifts, this location will be where $\kappa_{j}=0$ ). Assume that the matched envelope functions satisfying the KV equation (1) are symmetric about the mid-drift with

$$
r_{j}(s)=r_{\tilde{j}}(-s) .
$$

Here, if $j=x, y$, then $\tilde{j}=y, x$. Take the $j=x \mathrm{KV}$ equation [see Eq. (1)], substitute $s \rightarrow-s$. Then employing the focusing and envelope symmetries in Eqs. (A1) and (A2) together with $\kappa_{y}=-\kappa_{x}$ obtains the complementary $j=y$ $\mathrm{KV}$ equation, thereby showing that the assumed symmetry in Eq. (A2) is consistent. An immediate corollary of Eq. (A2) is that at any mid-drift between quadrupoles, the envelope is round (i.e., $r_{x}=r_{y}$ ) with opposite convergence angles (i.e., $r_{x}^{\prime}=-r_{y}^{\prime}$ ).

Restrict the situation described above to a symmetric FODO system where the focusing and defocusing quadrupoles of the doublet are separated by equal length axial drifts [3] and the focusing and defocusing elements are each reflection symmetric about their axial midpoint [i.e., within one element, $\kappa_{x}(s-\tilde{s})=\kappa_{x}(-s+\tilde{s})$ where $s=\tilde{s}$ is the geometric field center of the element]. These further assumptions lead to the additional FODO focusing symmetry

$$
\kappa_{j}(s)=\kappa_{\tilde{j}}\left(L_{p} / 2+s\right) .
$$

With the choice of $s=0$ made as above, the focusing and defocusing optical elements are centered at $s=L_{p} / 4$ and $s=3 L_{p} / 4$ within the period $s \in\left[0, L_{p}\right]$. Using steps analogous to those outlined above, it can be shown that the matched envelope functions also have the FODO symmetry:

$$
r_{j}(s)=r_{\tilde{j}}\left(L_{p} / 2+s\right)
$$

Another FODO symmetry can be obtained by replacing $s \rightarrow-s$ in Eq. (A4), applying Eq. (A2), and differentiating to yield

$$
r_{j}^{\prime}(s)=-r_{j}^{\prime}\left(L_{p} / 2-s\right)
$$

Evaluating this expression at the focusing element centers at $s=L_{p} / 4$ and $s=3 L_{p} / 4$ and invoking periodicity of the $r_{j}$ with $r_{j}^{\prime}\left(s+L_{p}\right)=r_{j}^{\prime}(s)$ shows that the matched envelope functions are extremized (i.e., $r_{j}^{\prime}=0$ ) at the focusing element centers in a symmetric FODO lattice. The envelope equation (1) then shows that the $j$-plane extrema of $r_{j}$ with $\kappa_{j}<0$ (defocusing plane) satisfies $r_{j}^{\prime \prime}>$ 0 and therefore must be a minimum value. Period symmetries then require that the other focusing plane extrema $\left(\tilde{j}\right.$-plane with $\left.\kappa_{\tilde{j}}>0\right)$ corresponds to a maximum value.

Analogous steps to those employed in the analysis of quadrupole doublet focusing can be applied to solenoidal focusing $\left(\kappa_{x}=\kappa_{y}\right)$ systems with $\varepsilon_{x}=\varepsilon_{y}$ to show that 
$r_{x}=r_{y}$. Consider a periodic solenoidal focusing function with only a single element in the period that is also reflection symmetric about the axial midplane (with reflection symmetry defined as for the FODO quadrupole case above). Then procedures used above are readily employed to show that the matched envelope function $r_{j}$ is maximum at the axial center of the focusing element and is minimum at the axial center of the drift.

[1] I. Kapchinskij and V. Vladimirskij, in Proceedings of the International Conference on High Energy Accelerators and Instrumentation (CERN Scientific Information Service, Geneva, 1959), p. 274.

[2] M. Reiser, Theory and Design of Charged Particle Beams (John Wiley \& Sons, Inc., New York, 1994).

[3] S. M. Lund and B. Bukh, Phys. Rev. ST Accel. Beams 7, 024801 (2004).

[4] R. Ryne, Technical Report acc-phys/9502001, http:// arxiv.org, Accelerator Physics (1995).

[5] H. Wiedemann, Particle Accelerator Physics: Basic Principles and Linear Beam Dynamics (Springer-Verlag, New York, 1993).

[6] E. P. Lee, Part. Accel. 52, 115 (1996).

[7] E. P. Lee, Phys. Plasmas 9, 4301 (2002).
[8] O. Anderson, in Proceedings of the 2005 Particle Accelerator Conference, Knoxville, TN (IEEE, Piscataway, NJ, 2005), p. TPAT061.

[9] O.A. Anderson (to be published).

[10] R. C. Davidson, Physics of Nonneutral Plasmas (AddisonWesley, Reading, MA, 1990), rereleased, World Scientific, 2001.

[11] I. Hofmann, L. J. Laslett, L. Smith, and I. Haber, Part. Accel. 13, 145 (1983).

[12] E. D. Courant and H. S. Snyder, Ann. Phys. (Leipzig) 3, 1 (1958).

[13] E. Lee and R. J. Briggs, Technical Report LBNL-40774, UC-419, Lawrence Berkeley National Laboratory, 1997.

[14] R.C. Davidson and Q. Qian, Phys. Plasmas 1, 3104 (1994).

[15] R. C. Davidson and H. Qin, Physics of Intense Charged Particle Beams in High Energy Accelerators (World Scientific, New York, 2001).

[16] S. Wolfram, The Mathematica Book (Wolfram Media, Champaign, IL, 2003), 5th ed.

[17] S. M. Lund, S. H. Chilton, and E. P. Lee, http://arxiv.org/ abs/physics/0602150.

[18] See EPAPS Document No. E-PRABFM-9-005606 for programs and supplementary material. For more information on EPAPS, see http://www.aip.org/pubservs/ epaps.html. 